Article

\title{
Spatial Variations of Bacterial Communities of an Anaerobic Lagoon-Type Biodigester Fed with Dairy Manure
}

\author{
Marleny García-Lozano ${ }^{1}$, Inty Omar Hernández-De Lira ${ }^{1}$, David H. Huber ${ }^{2}$ and \\ Nagamani Balagurusamy ${ }^{1, *(i)}$ \\ 1 Laboratorio de Biorremediación, Facultad de Ciencias Biológicas, Universidad Autonoma de Coahuila, \\ Torreon 27000, Mexico \\ 2 Department of Biology and Gus R. Douglas Institute, West Virginia State University, \\ Institute, WV 25112, USA \\ * Correspondence: bnagamani@uadec.edu.mx; Tel.: +52-871-757-1785
}

Received: 17 April 2019; Accepted: 26 June 2019; Published: 1 July 2019

\begin{abstract}
Anaerobic digestion technology is being widely employed for sustainable management of organic wastes generated in animal farms, industries, etc. Nevertheless, biodigester microbiome is still considered a "black box" because it is regulated by different physico-chemical and operational factors. In this study, the bacterial diversity and composition in different sites of a full-scale lagoon type biodigester $\left(23,000 \mathrm{~m}^{3}\right)$ fed with dairy manure, viz., the influent, beginning, middle, final and effluent were analyzed. The biodigester registered a total of 1445 OTUs, which demonstrated the complex microbial ecosystem in it. Of them, only six OTUs were shared among all the different sampling points. The most abundant phyla belonged to Firmicutes, Proteobacteria, Latescibacteria and Thermotogae. The Simpson and Shannon index showed that the highest microbial diversity was observed in the beginning point of the biodigester, meanwhile, the lowest diversity was recorded in the middle. Based on the UniFrac distances, microbial communities with high similarity were recorded in the middle and final of the biodigester. It can be clearly observed that bacterial communities varied at the different points of the biodigester. However, based on metagenome predictions using PICRUSt, it was found that independent of the differences in taxonomy and location, bacterial communities maintained similar metabolic functions.
\end{abstract}

Keywords: anaerobic digestion; biodigester; bacterial communities; PICRUSt; spatial variations

\section{Introduction}

Anaerobic digestion (AD) is a complex process in which organic matter is degraded by different microbial communities to produce biogas. This process is applied for the treatment of a wide range of organic wastes from agriculture, livestock, industries and municipalities. In this regard, it should be highlighted that the agriculture sector represents an important source of greenhouse gas emissions (GHGs), contributing to 10-12\% of the total global anthropogenic GHG emissions [1] and of which, livestock contributes to $19 \%$ of total GHG emissions [2]. Hence, it is important to develop sustainable technologies to minimize GHG emissions and as well to recover energy from these resources, which in general are considered as wastes. AD offers sustainable management of cattle manure coupled with renewable energy production in the form of methane, which can be used to generate heat and electricity since has a calorific value of 21-24 MJ $/ \mathrm{m}^{3}$ [3]. The use of biodigesters for the treatment of animal manures has increased over the past 70 years and is wide-spread around the world. Potential of bioenergy in Mexico by the anaerobic treatment of animal manures has been estimated to be 410.41 GWh of electricity reducing methane emissions by $2240.64 \mathrm{Gg} \mathrm{CO}_{2}$ Eq [4]. In Mexico, more than 1000 
lagoon-type anaerobic biodigesters have been installed for the treatment of these wastes [5]. These systems are anaerobic ponds with a capacity in the range of 300-30,000 $\mathrm{m}^{3}$ and are enclosed with an impermeable cover, where manure enters it and follows a horizontal flow through the lagoon until exiting as effluent. This type of biodigester is generally operated on Mexican dairy farms because of its simplicity, low cost and low maintenance requirements; even though, most of these biodigesters are not efficient in methane production since their operation is based on empirical knowledge without considering the microbiome present which plays an essential role in the biodigester performance. Metagenomic studies of microbial communities in biogas digesters have been widely reported [6-8]. Nevertheless, due to their complexity, these communities have not been yet precisely characterized and this process is still considered a "black box". In addition, the interaction of bacterial populations at different sites of a lagoon type biodigester is not studied yet. Knowledge on microbial community structure and interrelationships is essential to understand their function in organic matter degradation and consequently, the performance of biodigester.

Recently, in Comarca Lagunera anaerobic digesters have been applied as a strategy to treat dairy manure and mitigate the environmental impact of these organic wastes by producing clean energy in the form of methane. In Comarca Lagunera, there are about 300 farms and around 80-100 biodigesters have been installed. But only a fraction out of these biodigesters is working properly. More than $80 \%$ of the biodigesters are not working to their potential in biogas production and there are no case studies done which can help us to understand the problems and develop adequate strategies to recover the energy present in these wastes in the form of methane. Hence, it is proposed to evaluate the spatial variations of bacterial populations in a full-scale, good performance, lagoon type biodigester fed with dairy manure and operated in a dairy farm at Comarca Lagunera region of Mexico. Analyses of metagenome functional prediction were performed to obtain a better understanding of the community and their contribution to the functioning of the biodigester working under properly performance operation.

\section{Materials and Methods}

\subsection{Sample Collection}

The samples were collected from a dairy manure fed lagoon type biodigester $\left(23,000 \mathrm{~m}^{3}\right)$ located in the Comarca Lagunera region of Mexico in February with an average methane production of $60 \%$. The biodigester is operated under environmental temperature conditions with a retention time of 60 days without any mixing system. Samples from different point of the biodigester such as influent, beginning, middle, final and effluent point (see Figure 1) were collected by triplicate in sterile vials, a flexible tube was used to sample at a depth of $5 \mathrm{~m}$ from the slurry level at points 2, 3 and 4 (see Figure 1 from the top, then samples were store at $-20{ }^{\circ} \mathrm{C}$ until further processing. Genomic DNA was extracted using the PowerSoil DNA isolation kit (MO BIO Laboratories, Inc., Carlsbad, CA, USA), quantified by Nanodrop spectrophotometer (model \#ND-1000, Thermo Fisher Scientific, Inc., Whaltham, MA, USA) and was visualized by using agarose $(0.8 \%)$ gel electrophoresis.

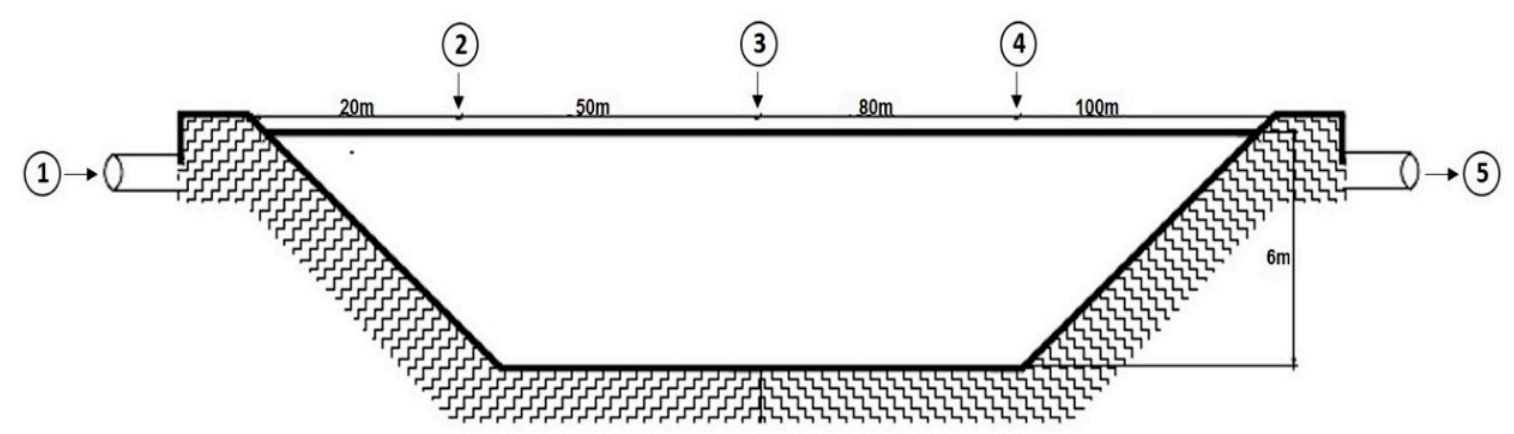

Figure 1. Scheme of the lagoon type biodigester. Cross-sectional view showing the sampling sites. Influent (1), beginning (2), middle (3), final (4) and effluent (5). 


\subsection{Microbial Community Composition by 454 Pyrosequencing}

The V4 region of the 16S rRNA gene of bacteria was employed for sequencing. One forward (5'-AYTGGGYDTAAAGNG-3') and three reverse primers (5'-TACNVGGGTATCTAATCC- ${ }^{\prime}$, $5^{\prime}$-TACCERGGGTHTCTAATCC-3' and $5^{\prime}$-CTACDSRGGTMTCTAATC- $3^{\prime}$ ) were used for pyrosequencing at ratio of 12:6:1, as recommended by Sul et al. [9]. The PCR mixture consisted of the following reagents, $5 \mu \mathrm{L}$ of 10X PCR buffer; $1 \mu \mathrm{L}$ of dNTP's mix; $0.5 \mu \mathrm{L}$ FastStart HiFi polymerase $(5 \mathrm{U} / \mu \mathrm{L}) ; 2 \mu \mathrm{L}$ of each forward and reverse primers; genomic DNA (40 ng) and molecular grade water to complete a final volume of $50 \mu$. The PCR reaction was carried out in the following conditions: 94 ${ }^{\circ} \mathrm{C}$ initializing temperature for $2 \mathrm{~min}, 30$ cycles of $94^{\circ} \mathrm{C}$ for $45 \mathrm{~s}, 57^{\circ} \mathrm{C}$ for $45 \mathrm{~s}$ and $72{ }^{\circ} \mathrm{C}$ for $1 \mathrm{~min}$, followed by a final extension of $72{ }^{\circ} \mathrm{C}$ for $4 \mathrm{~min}$. The reactions were run in triplicate and analyzed in a $1.2 \%$ agarose gel (1X TAE) at $110 \mathrm{~V}$ for $45 \mathrm{~min}$. The amplicons were excised from the gel (bands of $\sim 300$ $\mathrm{bp}$ ) using the Qiagen gel extraction Kit; triplicates were combined and purified with the QIAquick Gel Extraction Kit (QIAGEN, Inc., Valencia, CA, USA) and the amplicon extracted was verified by $0.8 \%$ agarose gel for $35 \mathrm{~min}$ at $85 \mathrm{~V}$. Equimolar concentrations of amplicons from each sample were pooled together and sequenced on a Roche 454 GS Junior system (Roche Applied Science, Indianapolis, IN, USA) at laboratory of the Department of Biology, West Virginia State University, USA.

Sequences were analyzed by QIIME pipeline version 1.7.0 [10]. Multiplexed reads were assigned to the samples based on a specific sequence of 10-bp (barcode). Sequences shorter than $100 \mathrm{bp}$ or longer $1000 \mathrm{bp}$ and with a quality score lower than 25 were removed. De novo OTU picking from quality-filtered reads was based on phylotype threshold of $97 \%$ sequence similarity. Reads were aligned using the Phyton Nearest Alignment Space Termination (PyNAST) algorithm [10] against the chimera-checked 16 S rRNA gene database, Greengenes. Taxonomy from the domain-level up to genus-level was assigned to each OTU using the Ribosomal Database Project Classifier algorithm at minimum confidence level of 0.8 [11].

\subsection{Diversity Analysis}

Alpha diversity metrics were calculated by the package phyloseq of $\mathrm{R}$ software [12] using as input the OTU table generated by QIIME. The metrics obtained were: observed species (richness), expected species (Chao1), the Simpson's dominance index and finally the Shannon's equality index. Rarefaction curves were constructed by the package iNEXT of R software [13]. For beta diversity analysis, dissimilarity matrices between samples were calculated in QIIME based on weighted and unweighted UniFrac distances, which were further visualized by Principal Coordinate Analyses (PCoA). UniFrac method allowed the comparison of the microbial communities present in the different sampling points based on their phylogenetic information in order to determine whether these communities were significantly different.

\subsection{Functional Analyses}

The functional role of microbial communities recorded in the biodigester was predicted from $16 \mathrm{~S}$ rRNA gene data using Phylogenetic Investigation of Communities by Reconstruction of Unobserved States (PICRUSt) software [14]. PICRUSt uses a computational approach to link the taxonomic assignments based on marker gene data to phylogenetically nearest sequenced reference genomes. PICRUSt compatible OTU table was obtained by closed-reference OTU picking protocol in QIIME against Greengenes (V. 12.2). Due to $16 \mathrm{~S}$ rRNA copy number varies among bacteria, OTU table was normalized dividing each OTU by the $16 \mathrm{~S}$ copy number abundance Normalization of OTU table by copy number was performed dividing each OTU by the $16 \mathrm{~S}$ copy number abundance. Metagenomic functions were predicted using the Kyoto Encyclopedia of Genes and Genomes (KEGG) databases for annotations, collapsed down to KEGG pathways metadata at level 2 and 3. The final metagenome functional predictions were obtained by the product of each OTU abundance and the abundance of each predicted functional trait. 


\subsection{Nucleotide Sequences Accession Numbers}

This Targeted Locus Study project has been deposited at DDBJ/EMBL/GenBank under the accession KBTP00000000. The version described in this paper is the first version, KBTP01000000.

\section{Results}

In this study, microbial communities from five different points of a biodigester (see Figure 1) were characterized using the $16 \mathrm{~S}$ rRNA gene in order to identify the spatial variations of bacterial groups and to relate their metabolic capacity and interactions with the different stages of the anaerobic digestion process.

\subsection{Bacterial Community Composition}

From the total sequences, 41 phyla, 79 classes, 112 orders, 124 families and 137 genera were observed. The most abundant phyla among all the samples were Firmicutes (33.91\%), followed by Proteobacteria (21.44\%), Latescibacteria $(6.82 \%)$, Thermotogae $(6.83 \%)$ and Synergistetes $(6.37 \%)$. As minor phyla Spirochaetes (3.31\%), Hyd24-12 (2.94\%), Lentisphaerae (1.93\%), Chloroflexi (1.92\%), Verrucomicrobia (1.92\%) and Acidobacteria (1.90\%) were presented. Unclassified bacteria, sequences that could not be assigned to specific phyla, contributed to $5.35 \%$ of total samples (see Figure 2).

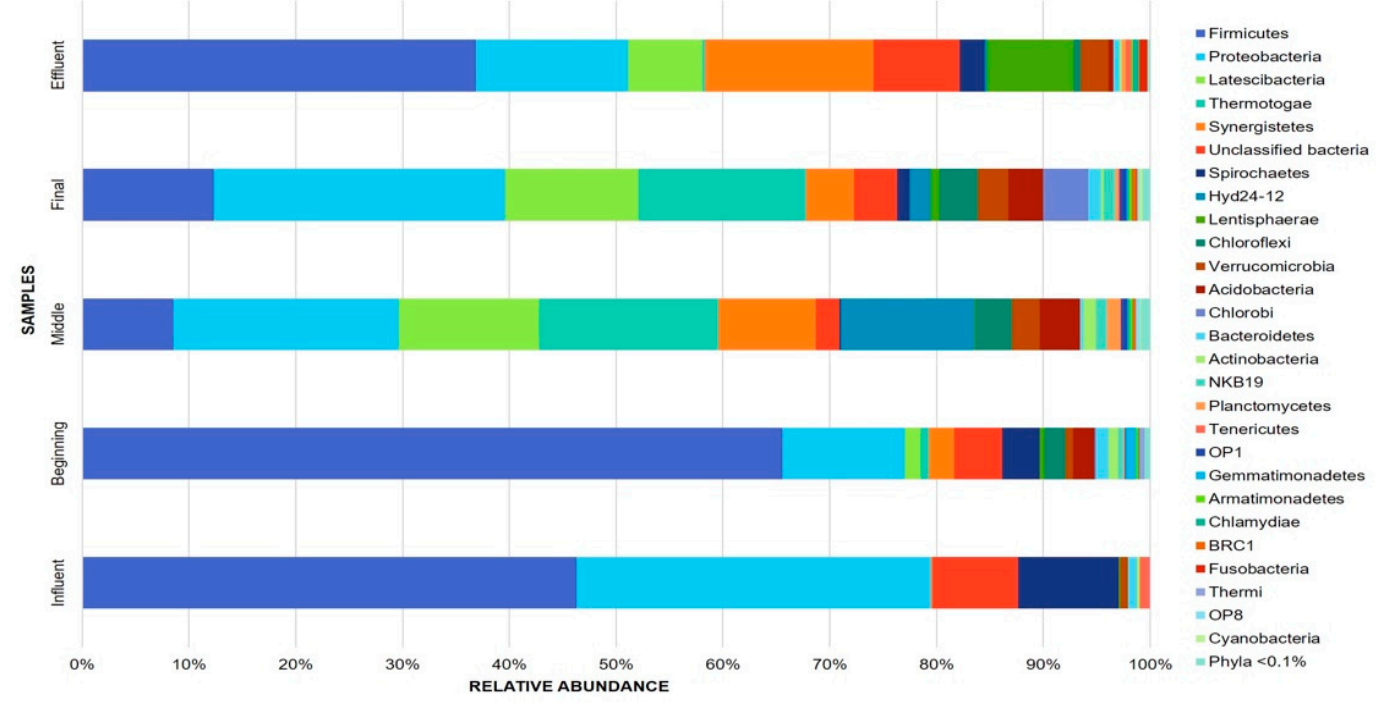

Figure 2. Relative abundance at phylum level among the different sampling sites of the biodigester.

Firmicutes $(46.28 \%)$, Proteobacteria $(33.2 \%)$ and Spirochaetes $(9.47 \%)$ were dominant in the influent. Additionally, these phyla were also preeminent in the beginning, differing only in microbial abundance. Nonetheless, microbial communities in these sites variated at lower taxonomic levels. At phylum level, the most remarkable change in the beginning compared to the influent was the decrease of Spirochaetes $(3.51 \%)$, which not only declined at this site but also at different sampling sites of the biodigester.

Minor differences between microbial communities at the phylum level were also observed between middle and final sampling points. Whereas Proteobacteria $(21.09 \%)$, Thermotogae $(16.80 \%)$, Latescibacteria (13.16\%) and Hyd24-12 (12.5\%) were observed to be the top four phyla in the middle of the biodigester, the most abundant phyla in the final site were Proteobacteria (27.25\%), Thermotogae (15.68\%), Firmicutes (12.33\%) and Latescibacteria (12.51\%).

In the effluent site Firmicutes (33.88\%), Synergistetes (15.74\%), and Proteobacteria (14.22\%) where the most abundant phyla. It is important to mention that Synergistetes and Lentisphaerae $(8.13 \%)$ 
abundances were considerably higher in this location in comparison with the other sites of the biodigester, meanwhile, Thermotogae decreased drastically in this site.

The most abundant orders also showed the same pattern in all sampling points of the biodigester. They belonged to Clostridiales $(25.40 \%)$, followed by Syntrophobacterales $(6.89 \%)$, Unclassified Latescibacteria $(6.73 \%)$, Thermotogales $(6.71 \%)$, Synergistales $(6.36 \%)$ and Coriobacteriales $(3.07 \%)$ (see Figure 3). In the influent site, Clostridiales (37.23\%), Pseudomonales (14.47\%) and Aeromonadales $(9.74 \%)$ and Spirochaetales $(9.45 \%)$ were the most prevalent orders. Clostridiales $(47.67 \%)$ also presented a high relative abundance in the beginning and in addition with Coriobacteriales $(10.44 \%)$ and Synergistales $(2.34 \%)$, these were the top three orders in this sample. Middle and Final sites repeatedly exhibited a similar community structure being Thermotogales (16.8-15.68\%), Syntrophobacterales (16.32-14.28\%) and Unclassified Latescibacteria (13.01-12.36\%) the dominant orders in these sampling points. Microbial population in the effluent consisted mostly of Clostridiales (29.64\%), Synergistales $(15.74 \%)$ and Z20 (7.61\%).

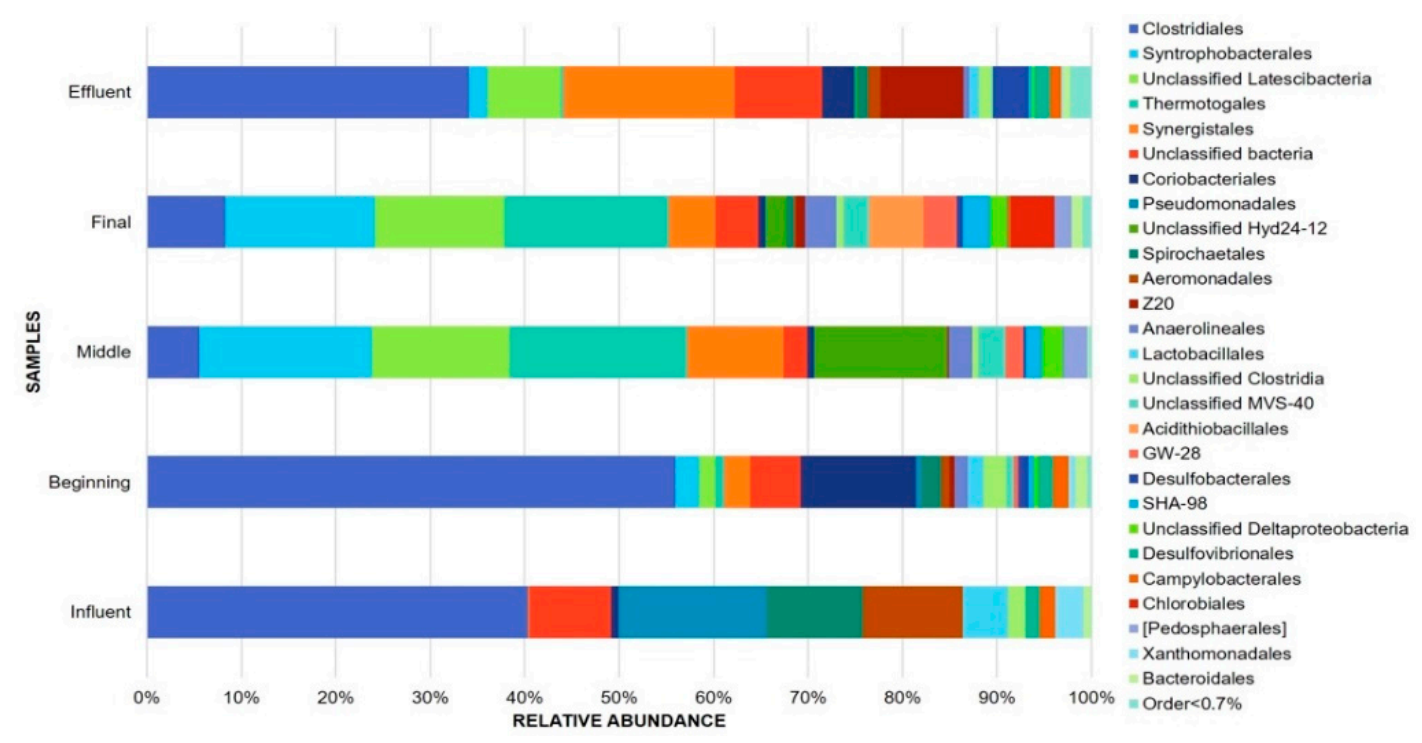

Figure 3. Relative abundance at order level among the different sampling sites of the biodigester.

\subsection{Alpha Diversity}

A total of 20,852 high-quality sequences were obtained with a mean of 4170 sequences per sample. These sequences were clustered into 1445 bacterial operational taxonomic units (OTUs), including 377 singleton OTUs. Rarefaction curves for almost all the samples presented an asymptotic behavios and the observed OTUs were very similar to the expected OTUs calculated by Chao1 demosntrating the sufficient sampling effort in this study Chao1 (see Figures S1 and S2 in Supplementary Materials).

Overall, the influent sample presented the lowest richness in comparison with the other sampling sites (326 OTUs). Upon introduction from the beginning of the biodigester recorded 549 OTUs, being the site with the highest richness and additionally, the most diverse according to Shannon and Simpson index (see Figure S2 in Supplementary Materials).

\subsection{Beta Diversity}

Beta diversity was calculated to compare the divergence of the sequences among the different sampling points of the biodigester based on UniFrac distances. While weighted UniFrac distance considers the relative abundance of the microbial population, unweighted UniFrac distance is calculated based only in presence/absence [15]. Results of both distances showed similar results (see Figure 4a,b), indicating that the community structure was similar in the middle and the final as well as in the beginning and the effluent. In addition, the influent had the most distinct microbial composition according to both distances. 


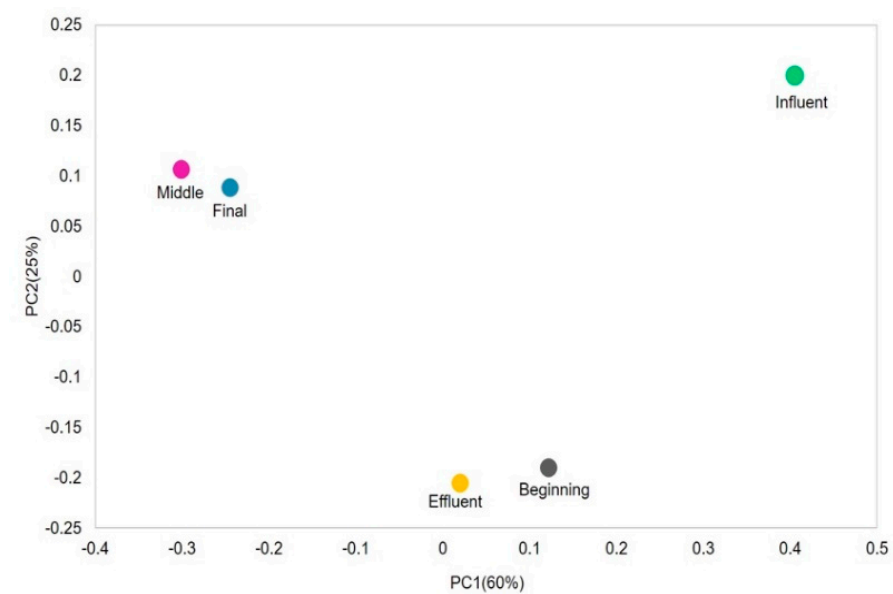

(a)

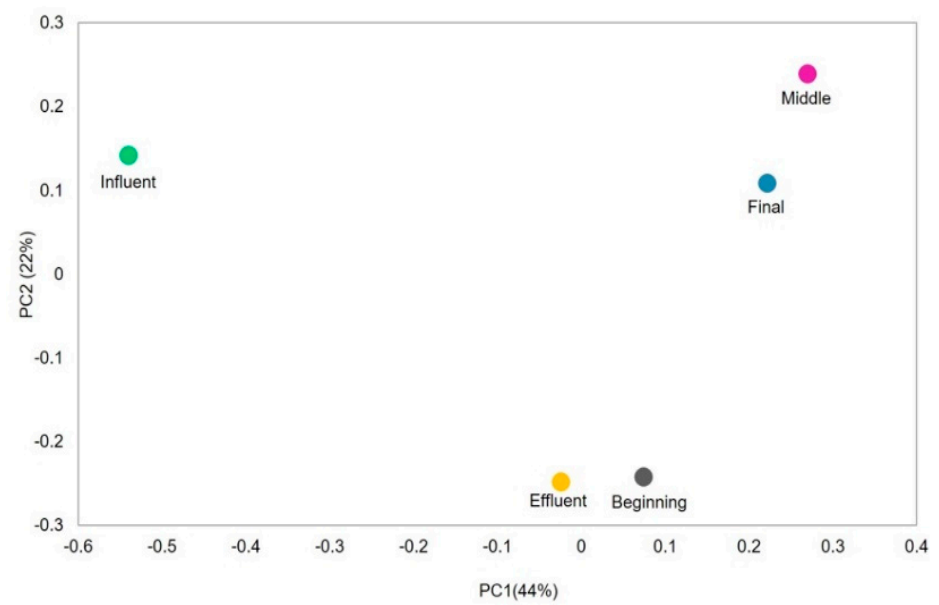

(b)

Figure 4. (a) Principal Coordinate Analyses (PCoA) plot based on weighted UniFrac distance between the different sampling sites of the biodigester. The percentage variation explained with first two principal components (P1, P2); (b) Principal Coordinate Analyses (PCoA) plot based on unweighted UniFrac distance between the different sampling sites of the biodigester. The percentage variation is explained with first two principal components (P1, P2).

\subsection{Functional Profile of Microbial Communities}

The functional profile of microbial communities from all the samples was predicted from $16 \mathrm{~S}$ rRNA gene data by using PICRUSt. The most dominant metabolic functions in the biodigester were related to central metabolisms including "Amino Acid Metabolism", "Carbohydrate Metabolism" and "Energy Metabolism" (see Figure 5 and Figure S5 in Supplementary Materials). Carbohydrate metabolism included Amino sugar and nucleotide sugar metabolism, Glycolysis/Gluconeogenesis, Pyruvate metabolism and Citrate Cycle. The most abundant pathways related to amino acid metabolism were "Amino acid related enzymes", "Arginine and proline metabolism" and the "Alanine, aspartate and glutamate metabolism" (see Figures S3 and S4 in Supplementary Materials). It was observed that most of the metabolic functions were less abundant in the influent compared to the other sites of the biodigester. 


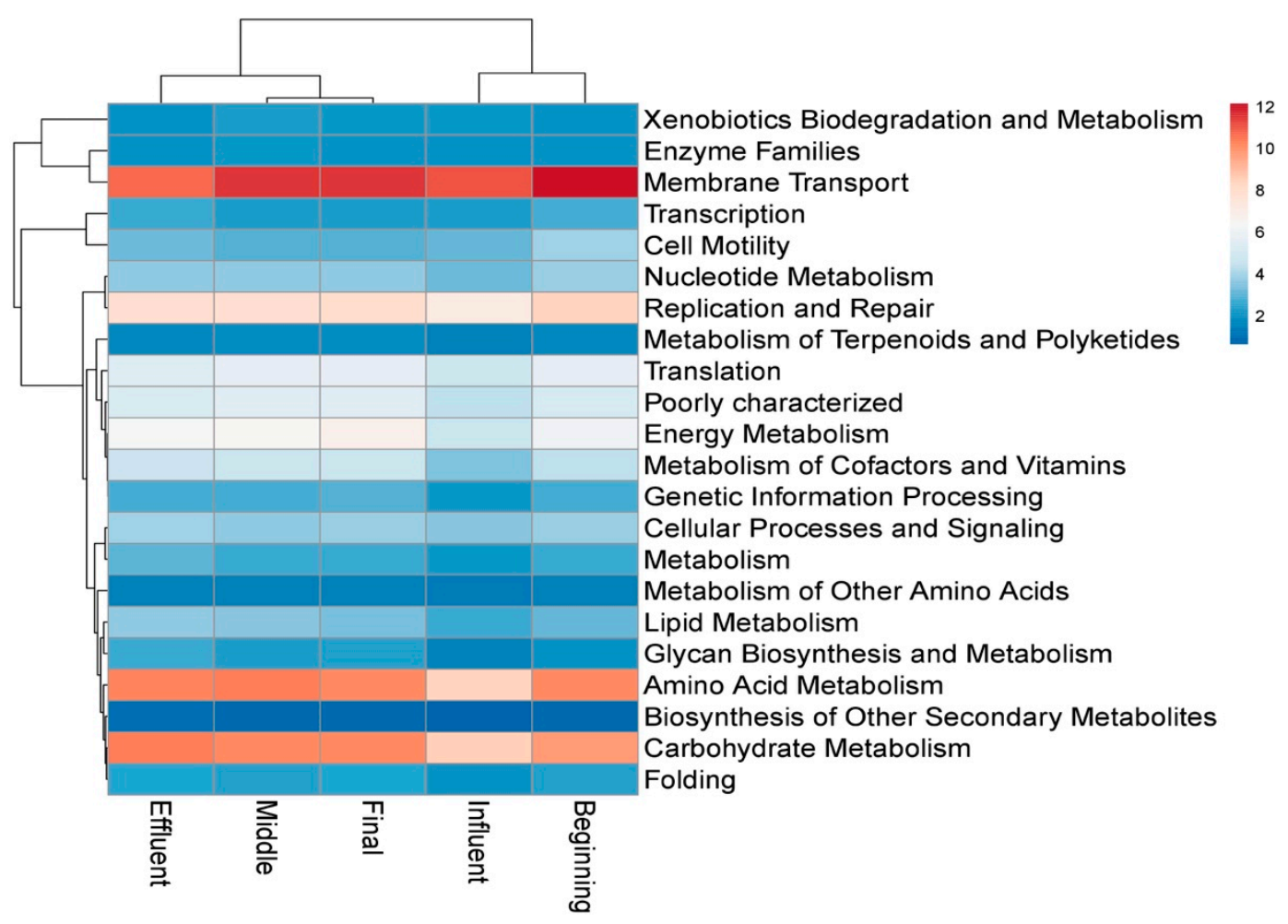

Figure 5. Heatmap of the functional prediction by PICRUSt at five sampling sites.

\section{Discussion}

Results of this study demonstrated a diverse and complex microbiome in the lagoon type biodigester. The number of OTUs obtained in this study is higher in comparison with other reports on microbial diversity of anaerobic digesters [6-8]. Further, microbial communities varied among the different sampling sites of the biodigester. This variability could be attributed to the operation of this biodigester which creates a gradient of unique conditions through the sampling points such as substrate availability, redox potential, $\mathrm{pH}$, Volatile Fatty Acids (VFA) concentration, etc. Earlier, Ye and Zhang [16], also reported variations in bacterial diversity in the different sections of a wastewater treatment plant. Nonetheless, in a study of different sites of a mixed plug-flow-loop reactor (MPFLR) fed with cattle manure, bacterial community was found homogeneous, and could be attributed to the mixing of the contents in the reactor [17]. Whereas no such mixing occurs in the lagoon-type biodigester of this present study, the prevalent environmental gradients resulted in heterogeneous bacterial communities in the biodigester.

The most abundant phyla, Firmicutes, Proteobacteria, Latescibacteria and Thermotogae, also have been reported as dominant in previous studies of biodigesters, albeit Latescibacteria has been described as minor phyla [18-20]. Firmicutes has been reported earlier in manure-based samples [8,21] and in several studies of anaerobic digesters [19,22-25]. The greater presence of Firmicutes, mainly represented by the cellulolytic and fermentative order Clostridiales, in the influent $(37.23 \%)$ and beginning sites $(47.67 \%)$ could be due to the high cellulose content of cattle manure, which because of the design of the reactor was present at high amounts in the first section of the biodigester [26]. This occurrence announces the major role of Clostridiales in the hydrolytic stage of anaerobic digestion process which might be predominantly performed in the influent and the beginning of the biodigester.

Influent site showed the most distinct microbial communities since some groups present in high abundance in this site were almost null in the other sections of the biodigester. These bacterial groups included Pseudomonales (14.47\%), Spirochetales (9.45\%), Aeromonadales (9.74\%), Lactobacillales $(4.44 \%)$ and Xanthomonadales $(2.77 \%)$ whose members have been recognized as pathogens for humans $[27,28]$. Our results suggest that their presence in the animal manure is normal, but the 
environmental conditions of the biodigester are not favorable to their prevalence and proliferation. Thus, AD process can be considered efficient regarding the elimination of pathogens. Lactobacillales predominance in the influent has been previously observed by Li et al. [17] Furthermore, the presence of these facultative bacteria along with Pseudomonales and Xanthomonadales may be explained by the exposure of the animal manure to oxygen at this sampling point. Fu et al. [29] reported that microaerobic conditions improved the abundance of Lactobacillales in laboratory-scale reactors using bioslurry and corn straw as substrate.

Different microbial communities were dominant in the middle and final stage sites compared to the entrance of the biodigester. In these sections, a steady state of the biodigester might be expected due to the presence of simple compounds resulting from the breakdown of the organic matter in the first parts of the biodigester. This led to shared microbial groups between the middle and final segments.

The high abundance of members of Proteobacteria in these locations could be attributed to their capability to perform a great variety of metabolic functions such as sulfate and nitrate co-reduction $[30,31]$. However, Proteobacteria decreased drastically in the effluent, which was also observed by Li et al. [17]. The behavior of Proteobacteria across the different points of the biodigester could be assigned as functionally redundant, since despite their abundance diminished through the sampling points of the biodigester, the predicted functional profile of the communities stayed the same.

A high abundance of Latescibacteria was observed in these points, where Thermotogae also was highly recorded. The candidate phylum Latescibacteria has been described as anaerobic fermentative bacteria based on genome in silico analysis [32]. This group has been previously reported in anaerobic digesters fed with rice straw and in slurry samples [33]. Thermotogae have been found in both mesophilic [34-36] and thermophilic conditions [36-38], more so in later conditions. This phylum was mostly represented by Thermotogales $(16.8 \%$ and $15.68 \%)$. This order has been affiliated to perform the syntrophic acetate oxidation in biogas digesters [39] and has been reported in thermophilic digestion of sludge and manure [7]. The high occurrence of thermophilic bacteria in the middle and final parts of the biodigester might suggest an increase in the temperature of the biodigester at these points. The presence of Syntrophobacterales (16.32-14.28\%) and Synergistales $(9.1-4.46 \%)$ at these sites might be a sign of being acetogenic since members of Syntrophobacterales perform the propionate and butyrate oxidation and Synergistales as Thermotogales, carry out the oxidation of acetate, completing the anaerobic degradation $[25,40]$.

Relative abundance of Clostridiales was increased at the effluent (29.64\%), these microbial groups were defined as resilient since they could recover almost to their original composition in the biodigester, which might be by growth, or by physiological or genetic adaptation [41].

Unique and shared microbial communities among the different samples is presented in Figure 6. The number of unique OTUs in each sample varied from 116 (effluent) to 260 (beginning). Six OTUs co-occurred at all the sites of the biodigester and belonged mainly to Clostridiales. The major proportion of Clostridiales order indicated higher hydrolytic and fermentative activity, which could be related to VFA formation. In addition, their activity facilitated the microbial interactions as other trophic groups in biodigesters are dependent upon the metabolites produced. 


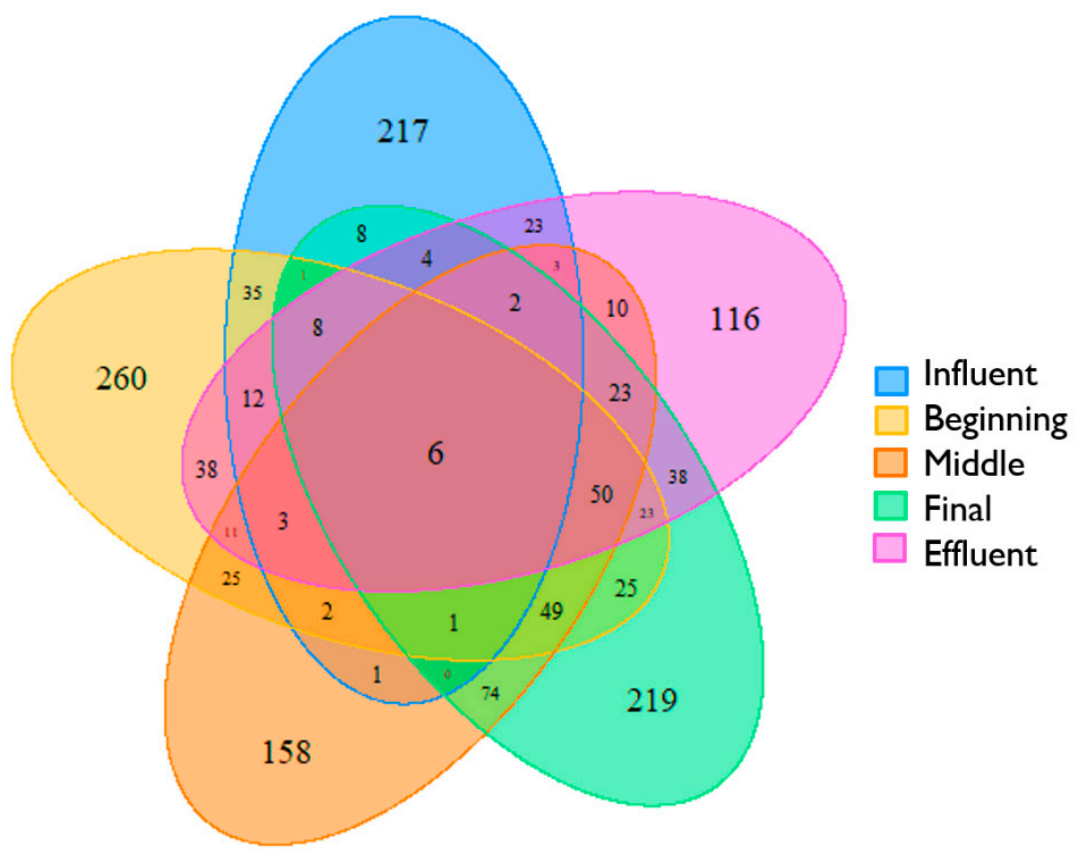

Figure 6. Venn diagram representing the shared OTUs between the different sampling sites of biodigester. Each sample is differentiated by the colors: influent (blue), beginning (yellow), middle (orange), final (green) and effluent (purple). The numbers show the OTUs recovered from that point.

The fact that the beginning sample presented the highest number of unique OTUs and the major diversity based on Shannon and Simpson indices might be attributed to the shift in microbial population from the manure storage to the entry of the biodigester due to the environmental transitions, which could have altered the microbial diversity to adapt to the existing conditions in the biodigester. Li et al. [17] also observed a high diverse bacterial community in the initial parts of the biodigester. This fact was explained by Loreau and Mazancourt [42] who described that communities introduce distinct species to achieve the stability in an ecosystem after disturbance.

It can be observed that middle and final sampling points shared major quantity of OTUs (74 OTUs). These samples also demonstrated a high similarity of bacterial diversity based on the relative abundance of the taxa at distinct levels and by the weighted and unweighted UniFrac PCoA. The final sampling point of the biodigester shared a major quantity of OTUs with the other sampling points, eight OTUs with the influent, 25 OTUs with the beginning, 74 OTUs with the middle and 38 OTUs with the effluent. Although the results of this study indicated that the microbial diversity varied at different stages of the AD process, high sharing of OTUs of the final site with other sites could be related to the presence of non-degraded monomers and intermediary products that are formed during the process and accumulated at the end of the biodigester due to their horizontal flow-through in the lagoon-type biodigester. Results of the predicted functional profile demonstrated the key pathways that takes place at different sampling sites of the biodigester.

Results of the predicted functional profile demonstrated the key pathways associated with the microbial communities present at different sampling sites of the biodigester. These pathways appeared related to the different stages of the anaerobic digestion process. Hydrolysis stage was represented by "Starch and sucrose metabolism" and by "Galactose", and "Fructose and mannose metabolism" since these monosaccharides are part of a wide variety of polysaccharides, although they also might be playing a role in the fermentation stage as well as "Butanoate metabolism", "Propanoate metabolism", "C5-Branched dibasic acid metabolism" and "Glycolysis". "Butanoate metabolism" and "Propanoate metabolism" might be also associated with acetogenesis stage, specifically in the catabolism of these compounds. 
Metabolic properties of the microbial communities differed by location indicated that AD is a dynamic process, and there are points where specific stages of $\mathrm{AD}$ can be more active through the biodigester. Our results revealed the role of the hydrolytic and fermentative metabolism of Firmicutes in the samples collected from influent and beginning. In addition, the diverse metabolism of Proteobacteria, mainly VFAs degradation and hydrogen consumption, was apparent in the middle and final sampling points. Moreover, the carbohydrate metabolism of Synergistetes was noticed in the effluent samples. Nonetheless, the results obtained by PICRUSt analyses showed that there were no differences in the functional profile of the microbial communities among the different points of the biodigester, suggesting that different microorganisms carried out similar functions independently of their location and taxonomy (see Figure 7). Gao et al. [23] also reported a similar result in the analysis of different samples of AD sludge.

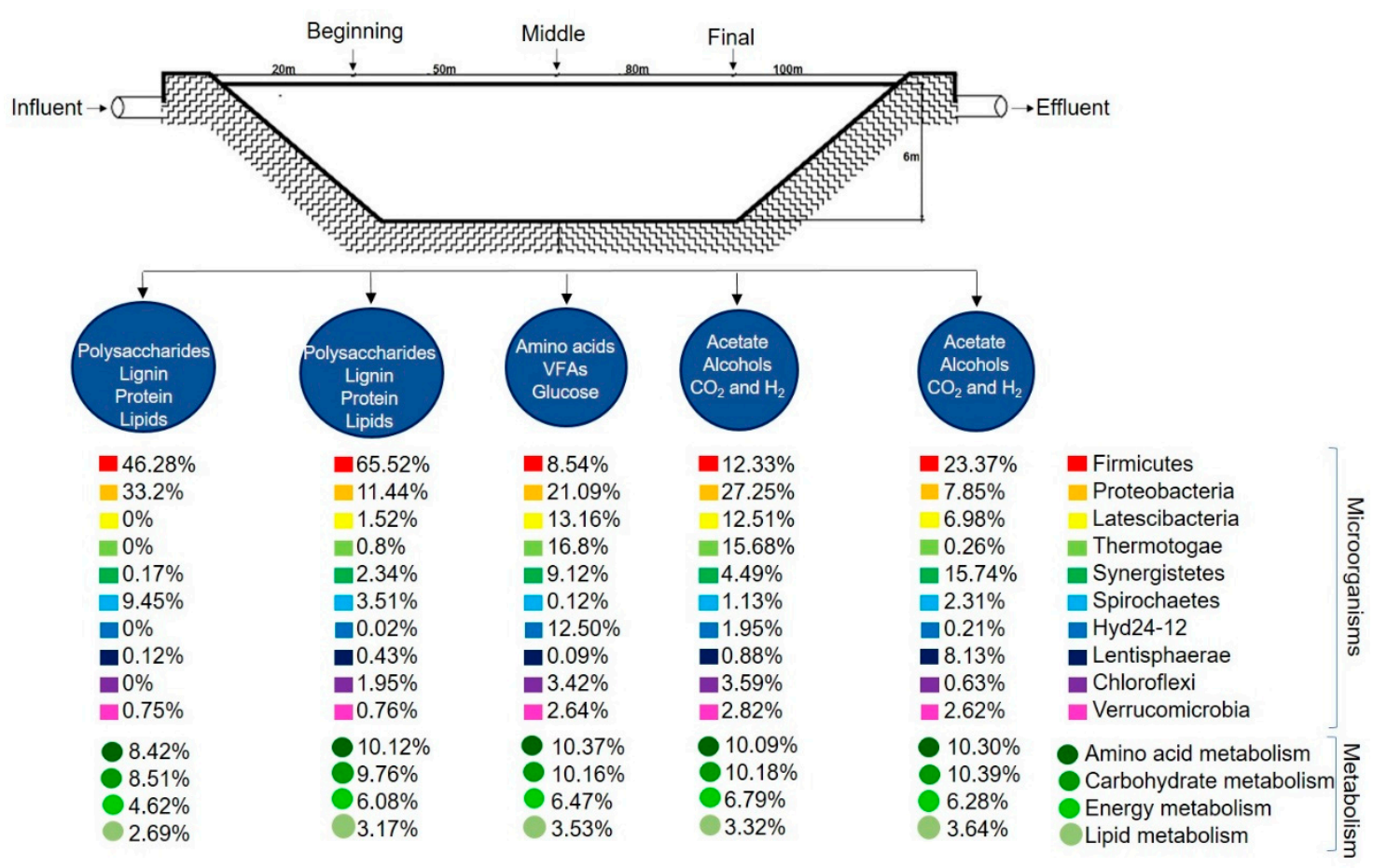

Figure 7. Overview of the bacterial population and metabolic pathways in the different sampling sites of the biodigester.

Distribution of specific taxa shifted in response to heterogenous conditions in order to allow the equilibrium and the success of methane production in the system. This phenomenon was noticed since a core of functional traits was observed through the biodigester.

\section{Conclusions}

The present study demonstrated that the diversity and abundance of bacterial communities varied among the different sampling points of the biodigester and was in accordance with the stages of the anaerobic digestion process. Of the total 1445 OTUs, only six OTUs were found to be shared among all the five sampling points. It was observed that the microbial diversity of the middle and final sampling sites was almost similar, meanwhile, influent was the most distinct point. Although AD is a dynamic process and different metabolic pathways could occur at the same sampling point, the differences in the abundance of microbial groups suggest that the bacterial group were related to specific metabolic process of AD. Conversely, based on the inference of PICRUSt, it can be observed that irrespective of the differences of the bacterial communities among the different sampling points of the biodigester, they maintained similar functions independent of their taxonomy. Microbial populations in this biodigester showed to be in a dynamic fashion maintaining the indispensable syntrophic 
conditions for methanogenesis to be performed. The findings of this study highlighted the importance of understanding the spatial variations of microbial groups and their interactions to fine tune the operational parameters of lagoon-type biodigester in order to improve their methane production. In addition, quantify relationships between community structure and function might be develop novel strategies to control, optimization and diagnosis of biodigesters in Comarca Lagunera region, in order to take advantage of dairy manures to produce bioenergy in form of methane.

Supplementary Materials: The following are available online at http://www.mdpi.com/2227-9717/7/7/408/s1, Figure S1: Comparison of diversity between the different sampling points of the biodigester using different measures of alpha diversity, (a) Observed OTUs, (b) Chao1 index, (c) Shannon index and (d) Simpson index; Figure S2: Rarefaction curves for 16 S rRNA gene sequences derived from the biodigester (OTUs are retrieved at $97 \%$ of similarity); Figure S3: Prediction of functional profiling by abundances of KEGG pathways in level-3 (Amino acid metabolism) by using PICRUSt; Figure S4: Prediction of functional profiling by abundances of KEGG pathways in level-3 (Carbohydrate metabolism) by using PICRUSt; Figure S5: Abundances of KEGG pathways in level-2 of the functional prediction by PICRUSt.

Author Contributions: Conceptualization, N.B.; Data curation, I.O.H.-D.L.; Formal analysis, M.G.-L.; Funding acquisition, D.H.H. and N.B.; Investigation, M.G.-L., I.O.H.-D.L., D.H.H. and N.B.; Methodology, D.H.H. and N.B.; Resources, D.H.H. and N.B.; Supervision, D.H.H. and N.B.; Validation, M.G.-L., I.O.H.-D.L., D.H.H. and N.B.; Writing—original draft, M.G.-L.; Writing—review \& editing, M.G.-L., I.O.H.-D.L., D.H.H. and N.B.

Funding: This research was funded by CONACyT-SENER, project grant number S0019-2014-01-245113.

Acknowledgments: The authors would like to acknowledge the financial support provided from CONACyT-SENER through the project grant (S0019-2014-01-245113). IOHDL thank CONACyT PNPC for the fellowship received to complete his Master's degree in Biochemical Engineering.

Conflicts of Interest: The authors declare no conflict of interest.

\section{References}

1. Smith, P.; Martino, D.; Cai, Z.; O’Mara, F.; Rice, C.; Scholes, B.; Howden, M.; McAllister, T.; Pan, G.; Romanenkov, V.; et al. Agriculture. In Contribution of Working Group III to the Fourth Assessment Report of the Intergovernmental Panel on Climate Change; Cambridge University Press: Cambridge, UK, 2007; pp. 498-540.

2. Reisinger, A.; Clark, H. How much do direct livestock emissions actually contribute to global warming? Glob. Chang. Biol. 2018, 24, 1749-1761. [CrossRef] [PubMed]

3. Bond, T.; Templeton, M.R. History and future of domestic biogas plants in the developing world. Energy Sustain. Dev. 2011, 15, 347-354. [CrossRef]

4. Hernandez-De Lira, I.O.; Huber, D.H.; Espinosa-Solares, T.; Balagurusamy, N. Methane emission and bioenergy potential from livestock manures in Mexico. J. Renew. Sustain. Energy 2015, 7, 053117. [CrossRef]

5. Weber, B.; Rojas-Oropeza, M.; Torres-Bernal, M.; Pampillón-González, L. Produccion de Biogas en México, Estadp Actual y Perspectivas, 1st ed.; Red Mexicana de Bioenergia: Mexico city, Mexico, 2012; pp. 1-48.

6. Lee, J.; Han, G.; Shin, S.G.; Koo, T.; Cho, K.; Kim, W.; Hwang, S. Seasonal monitoring of bacteria and archaea in a full-scale thermophilic anaerobic digester treating food waste-recycling wastewater: Correlations between microbial community characteristics and process variables. Chem. Eng. J. 2016, 300, 291-299. [CrossRef]

7. Sun, W.; Yu, G.; Louie, T.; Liu, T.; Zhu, C.; Xue, G.; Gao, P. From mesophilic to thermophilic digestion: The transitions of anaerobic bacterial, archaeal, and fungal community structures in sludge and manure samples. Appl. Microbiol. Biotechnol. 2015, 99, 10271-10282. [CrossRef]

8. Sundberg, C.; Al-Soud, W.A.; Larsson, M.; Alm, E.; Yekta, S.S.; Svensson, B.H.; Sørensen, S.J.; Karlsson, A. 454 pyrosequencing analyses of bacterial and archaeal richness in 21 full-scale biogas digesters. FEMS Microbiol. Ecol. 2013, 85, 612-626. [CrossRef] [PubMed]

9. Sul, W.J.; Cole, J.R.; Jesus, E.D.C.; Wang, Q.; Farris, R.J.; Fish, J.A.; Tiedje, J.M. Bacterial community comparisons by taxonomy-supervised analysis independent of sequence alignment and clustering. Proc. Natl. Acad. Sci. USA 2011, 108, 14637-14642. [CrossRef]

10. Caporaso, J.G.; Kuczynski, J.; Stombaugh, J.; Bittinger, K.; Bushman, F.D.; Costello, E.K.; Fierer, N.; Peña, A.G.; Goodrich, J.K.; Gordon, J.I.; et al. QIIME allows analysis of high-throughput community sequencing data. Nat. Methods 2010, 7, 335-336. [CrossRef]

11. Wang, Q.; Garrity, G.M.; Tiedje, J.M.; Cole, J.R. Naive bayesian classifier for rapid assignment of rRNA sequences into the new bacterial taxonomy. Appl. Environ. Microbiol. 2007, 73, 5261-5267. [CrossRef] 
12. McMurdie, P.J.; Holmes, S. Phyloseq: An R package for reproducible interactive analysis and graphics of microbiome census data. PLoS ONE 2013, 8, e61217. [CrossRef]

13. iNEXT-Package: Interpolation and Extrapolation for Species Diversity in iNEXT: Interpolation and Extrapolation for Species Diversity. Available online: https://rdrr.io/cran/iNEXT/man/iNEXT-package.html (accessed on 9 April 2019).

14. Langille, M.G.I.; Zaneveld, J.; Caporaso, J.G.; McDonald, D.; Knights, D.; Reyes, J.A.; Clemente, J.C.; Burkepile, D.E.; Vega Thurber, R.L.; Knight, R.; et al. Predictive functional profiling of microbial communities using 16s rRNA marker gene sequences. Nat. Biotechnol. 2013, 31, 814-821. [CrossRef] [PubMed]

15. Lozupone, C.; Knight, R. UniFrac: A new phylogenetic method for comparing microbial communities. Appl. Environ. Microbiol. 2005, 71, 8228-8235. [CrossRef] [PubMed]

16. Ye, L.; Zhang, T. Bacterial Communities in different sections of a municipal wastewater treatment plant revealed by 16S rDNA 454 Pyrosequencing. Appl. Microbiol. Biotechnol. 2013, 97, 2681-2690. [CrossRef]

17. Li, Y.-F.; Chen, P.-H.; Yu, Z. Spatial and temporal variations of microbial community in a mixed plug-flow loop reactor fed with dairy manure. Microb. Biotechnol. 2014, 7, 332-346. [CrossRef] [PubMed]

18. Ziganshin, A.M.; Liebetrau, J.; Pröter, J.; Kleinsteuber, S. Microbial community structure and dynamics during anaerobic digestion of various agricultural waste materials. Appl. Microbiol. Biotechnol. 2013, 97, 5161-5174. [CrossRef]

19. Lee, S.H.; Kang, H.J.; Lee, Y.H.; Lee, T.J.; Han, K.; Choi, Y.; Park, H.D. Monitoring bacterial community structure and variability in time scale in full-scale anaerobic digesters. J. Environ. Monit. JEM 2012, 14, 1893-1905. [CrossRef] [PubMed]

20. Cardinali-Rezende, J.; Rojas-Ojeda, P.; Nascimento, A.M.A.; Sanz, J.L. Proteolytic bacterial dominance in a full-scale municipal solid waste anaerobic reactor assessed by 454 pyrosequencing technology. Chemosphere 2016, 146, 519-525. [CrossRef] [PubMed]

21. Luo, G.; Angelidaki, I. Analysis of bacterial communities and bacterial pathogens in a biogas plant by the combination of ethidium monoazide, PCR and Ion torrent sequencing. Water Res. 2014, 60, 156-163. [CrossRef]

22. Shin, S.G.; Koo, T.; Lee, J.; Han, G.; Cho, K.; Kim, W.; Hwang, S. Correlations between bacterial populations and process parameters in four full-scale anaerobic digesters treating sewage sludge. Bioresour. Technol. 2016, 214, 711-721. [CrossRef]

23. Gao, J.; Liu, G.; Li, H.; Xu, L.; Du, L.; Yang, B. Predictive functional profiling using marker gene sequences and community diversity analyses of microbes in full-scale anaerobic sludge digesters. Bioprocess Biosyst. Eng. 2016, 7, 1115-1127. [CrossRef]

24. St-Pierre, B.; Wright, A.D.G. Comparative metagenomic analysis of bacterial populations in three full-scale mesophilic anaerobic manure digesters. Appl. Microbiol. Biotechnol. 2014, 98, 2709-2717. [CrossRef] [PubMed]

25. Nelson, M.C.; Morrison, M.; Yu, Z. A meta-analysis of the microbial diversity observed in anaerobic digesters. Bioresour. Technol. 2011, 102, 3730-3739. [CrossRef] [PubMed]

26. Møller, H.B.; Moset, V.; Brask, M.; Weisbjerg, M.R.; Lund, P. Feces composition and manure derived methane yield from dairy cows: Influence of diet with focus on fat supplement and roughage type. Atmos. Environ. 2014, 94, 36-43. [CrossRef]

27. Naushad, H.S.; Gupta, R.S. Phylogenomics and molecular signatures for species from the plant pathogen-containing order Xanthomonadales. PLoS ONE 2013, 8, e55216. [CrossRef] [PubMed]

28. Martin-Carnahan, A.; Joseph, S.W. Aeromonadalesord. Nov. In Bergey's Manual ${ }^{\circledR}$ of Systematic Bacteriology: Volume Two the Proteobacteria Part B The Gammaproteobacteria; Brenner, D.J., Krieg, N.R., Staley, J.T., Garrity, G.M., Boone, D.R., De Vos, P., Goodfellow, M., Rainey, F.A., Schleifer, K.H., Eds.; Springer: Boston, MA, USA, 2005; pp. 556-587.

29. Fu, S.F.; He, S.; Shi, X.S.; Katukuri, N.R.; Dai, M.; Guo, R.B. The chemical properties and microbial community characterization of the thermophilic microaerobic pretreatment process. Bioresour. Technol. 2015, 198, 497-502. [CrossRef] [PubMed]

30. Chen, C.; Xu, X.J.; Xie, P.; Yuan, Y.; Zhou, X.; Wang, A.J.; Lee, D.J.; Ren, N.Q. Pyrosequencing reveals microbial community dynamics in integrated simultaneous desulfurization and denitrification process at different influent nitrate concentrations. Chemosphere 2017, 171, 294-301. [CrossRef] [PubMed] 
31. Chen, C.; Ren, N.; Wang, A.; Yu, Z.; Lee, D.J. Microbial community of granules in expanded granular sludge bed reactor for simultaneous biological removal of sulfate, nitrate and lactate. Appl. Microbiol. Biotechnol. 2008, 79, 1071-1077. [CrossRef]

32. Youssef, N.H.; Farag, I.F.; Rinke, C.; Hallam, S.J.; Woyke, T.; Elshahed, M.S. In silico analysis of the metabolic potential and niche specialization of candidate phylum "Latescibacteria" (WS3). PLoS ONE 2015, 10, e0127499. [CrossRef]

33. Zhou, J.; Yang, J.; Yu, Q.; Yong, X.; Xie, X.; Zhang, L.; Wei, P.; Jia, H. Different organic loading rates on the biogas production during the anaerobic digestion of rice straw: A pilot study. Bioresour. Technol. 2017, 244, 865-871. [CrossRef]

34. Li, A.; Chu, Y.; Wang, X.; Ren, L.; Yu, J.; Liu, X.; Yan, J.; Zhang, L.; Wu, S.; Li, S. A pyrosequencing-based metagenomic study of methane-producing microbial community in solid-state biogas reactor. Biotechnol. Biofuels 2013, 6, 3. [CrossRef]

35. Schlüter, A.; Bekel, T.; Diaz, N.N.; Dondrup, M.; Eichenlaub, R.; Gartemann, K.-H.; Krahn, I.; Krause, L.; Krömeke, H.; Kruse, O; et al. The metagenome of a biogas-producing microbial community of a production-scale biogas plant fermenter analysed by the 454-pyrosequencing technology. J. Biotechnol. 2008, 136, 77-90. [CrossRef] [PubMed]

36. Levén, L.; Eriksson, A.R.B.; Schnürer, A. Effect of process temperature on bacterial and archaeal communities in two methanogenic bioreactors treating organic household waste. FEMS Microbiol. Ecol. 2007, 59, 683-693. [CrossRef] [PubMed]

37. Pap, B.; Györkei, Á.; Boboescu, I.Z.; Nagy, I.K.; Bíró, T.; Kondorosi, É.; Maróti, G. Temperature-dependent transformation of biogas-producing microbial communities points to the increased importance of hydrogenotrophic methanogenesis under thermophilic operation. Bioresour. Technol. 2015, 177, 375-380. [CrossRef] [PubMed]

38. Pervin, H.M.; Dennis, P.G.; Lim, H.J.; Tyson, G.W.; Batstone, D.J.; Bond, P.L. Drivers of microbial community composition in mesophilic and thermophilic temperature-phased anaerobic digestion pre-treatment reactors. Water Res. 2013, 47, 7098-7108. [CrossRef] [PubMed]

39. Müller, B.; Sun, L.; Westerholm, M.; Schnürer, A. Bacterial community composition and fhs profiles of lowand high-ammonia biogas digesters reveal novel syntrophic acetate-oxidising bacteria. Biotechnol. Biofuels 2016, 9, 48. [CrossRef] [PubMed]

40. Regueiro, L.; Carballa, M.; Lema, J.M. Outlining microbial community dynamics during temperature drop and subsequent recovery period in anaerobic co-digestion systems. J. Biotechnol. 2014, 192, 179-186. [CrossRef]

41. Allison, S.D.; Martiny, J.B. Resistance, resilience, and redundancy in microbial communities. Proc. Natl. Acad. Sci. USA 2008, 105, 11512-11519. [CrossRef] [PubMed]

42. Loreau, M.; de Mazancourt, C. Biodiversity and ecosystem stability: A synthesis of underlying mechanisms. Ecol. Lett. 2013, 16, 106-115. [CrossRef]

(C) 2019 by the authors. Licensee MDPI, Basel, Switzerland. This article is an open access article distributed under the terms and conditions of the Creative Commons Attribution (CC BY) license (http://creativecommons.org/licenses/by/4.0/). 